\title{
Sharp acoustic vortex focusing by Fresnel-spiral zone plates
}

Noé Jiménez, Vicent Romero-García, Luis M. García-Raffi, Francisco Camarena, and Kestutis Staliunas

Citation: Appl. Phys. Lett. 112, 204101 (2018); doi: 10.1063/1.5029424

View online: https://doi.org/10.1063/1.5029424

View Table of Contents: http://aip.scitation.org/toc/apl/112/20

Published by the American Institute of Physics

\section{Articles you may be interested in}

Generation of fractional acoustic vortex with a discrete Archimedean spiral structure plate

Applied Physics Letters 112, 173501 (2018); 10.1063/1.5026646

Generation of multiple vortex beam by means of active diffraction gratings

Applied Physics Letters 112, 084101 (2018); 10.1063/1.5016864

Broadband unidirectional invisibility for airborne sound

Applied Physics Letters 112, 203502 (2018); 10.1063/1.5019771

Perspective: Acoustic metamaterials in transition

Journal of Applied Physics 123, 090901 (2018); 10.1063/1.5007682

Low-frequency and multiple-bands sound insulation using hollow boxes with membrane-type faces

Applied Physics Letters 112, 183506 (2018); 10.1063/1.5028547

Generation of radio vortex beams with designable polarization using anisotropic frequency selective surface Applied Physics Letters 112, 203501 (2018); 10.1063/1.5029507

\section{Conference Proceedings}




\title{
Sharp acoustic vortex focusing by Fresnel-spiral zone plates
}

\author{
Noé Jiménez, ${ }^{1, a)}$ Vicent Romero-García, ${ }^{2}$ Luis M. García-Raffi, ${ }^{3}$ Francisco Camarena, ${ }^{1}$ \\ and Kestutis Staliunas ${ }^{4}$ \\ ${ }^{1}$ I3M, Instituto de Instrumentación para Imagen Molecular, CSIC-Universitat Politècnica de València, \\ Valencia, Spain \\ ${ }^{2}$ Laboratoire d'Acoustique de la Université du Mans-CNRS UMR 6613, Le Mans, France \\ ${ }^{3}$ IUMPA, Universitat Politècnica de València, Camino de vers s/n, 46022 València, Spain \\ ${ }^{4}$ ICREA, Departament de Fisica i Enginyeria Nuclear, Universitat Politecnica de Catalunya, Colom 11, \\ E-08222 Terrassa, Barcelona, Spain
}

(Received 14 March 2018; accepted 3 May 2018; published online 14 May 2018)

\begin{abstract}
We report the optimal focusing of acoustic vortex beams by using flat lenses based on a Fresnelspiral diffraction grating. The flat lenses are designed by spiral-shaped Fresnel zone plates composed of one or several arms. The constructive and destructive interferences of the diffracted waves by the spiral grating result in sharp acoustic vortex beams, following the focal laws obtained in analogy with the Fresnel zone plate lenses. In addition, we show that the number of arms determines the topological charge of the vortex, allowing the precise manipulation of the acoustic wave field by flat lenses. The experimental results in the ultrasonic regime show excellent agreement with the theory and full-wave numerical simulations. A comparison with beam focusing by Archimedean spirals also showing vortex focusing is given. The results of this work may have potential applications for particle trapping, ultrasound therapy, imaging, or underwater acoustic transmitters. Published by AIP Publishing. https://doi.org/10.1063/1.5029424
\end{abstract}

Interaction of waves with matter is one of the most intriguing and most intensively studied phenomena in wave physics from the theoretical and applied viewpoints. The mechanical effects of waves on matter are related to the linear and angular momenta, two of the fundamental properties of the waves. Interestingly, wave fields with spiral phase dislocations, also called screw dislocations, ${ }^{1}$ carry orbital angular momentum (OAM) being able to create a torque associated with the transfer of angular momenta. In particular, helical or vortex beams, i.e., beams with a phase singularity on their axis, carry both linear and angular momenta offering unique possibilities for manipulating the matter. In optics, by exploiting these concepts, advanced technologies have been developed to design optical trapping, tweezing, sorting, chromatography, and rheology, among others. ${ }^{2}$ In acoustics, the transfer of OAM to solid objects by scattering or absorption leads to the generation of torques in macro-and microscopic objects. ${ }^{3-6}$ Moreover, vortex beams allow the generation of negative acoustic radiation forces on particles, and therefore, these kinds of acoustic vortices have recently received increasing interest mainly due to direct practical applications for particle trapping and manipulation. Acoustical tweezers ${ }^{7-9}$ or underwater transmitters ${ }^{10}$ have been designed based on these phenomena.

Vortex beams are characterized by a beam wavefront with a phase dependence of $e^{l l \phi}, \phi$ and $l$ being the azimuthal angle and the topological charge, respectively. The topological charge of the vortex is related to the efficiency on the momentum transfer. ${ }^{5}$ Therefore, in order to produce strong torques, the beam wavefront should be precisely focused conserving the screw dislocations of the vortex and controlling the topological charge. Acoustic vortices can be focused by using active devices as phase arrays where nonlinear

a)nojigon@upv.es propagation and vortex shock waves have been reported. ${ }^{11,12}$ However, the number of required independent active sources of the active system grows with the topological charge of the vortex, which increases the complexity of the system, its electronics, and its cost. Therefore, passive vortex generation techniques would be highly desirable in most applications. On the one hand, acoustic vortices have been reported using passive means by designing phase plates, ${ }^{13}$ exploiting the photo-acoustic effect, ${ }^{14}$ or by using helically deformed sources. ${ }^{15}$ On the other hand, metamaterials have been used to generate vortices by using flat and subwavelength structures, ${ }^{8,16-19}$ allowing a precise manipulation of the transmitted phase by using locally resonant inclusions.

Among these passive methods to excite acoustic vortices, the use of spiral diffraction gratings, in which the constructive and destructive interferences lead to the formation of a helical phase, has been revealed as a simple and efficient approach. $^{20,21}$ In the case of an Archimedes spiral, which presents a constant separation between slits, the diffracted field conforms a conical wavefront, leading to the formation of high-order Bessel beams. ${ }^{20} \mathrm{~A}$ diverging spiral, ${ }^{23}$ i.e., a spiral grating where the separation between slits increases with each winding, similar to the Lottus curve, also generates an acoustical vortex, but the energy is spread from the axis and the beam rapidly diverges. In addition, using active vibrating surfaces with spiral shape acoustic vortices can also be generated. $^{22}$ Moreover, beam focusing can be produced using axisymmetric diffraction gratings. Examples include the use of regularly spaced toroidal scatterers to obtain zero-order Bessel beams, ${ }^{24}$ optimized aperture gratings, ${ }^{25}$ or Fresnel zone plate (FZP) lenses. ${ }^{26-28}$

In this work, we introduce the concept of the Fresnelspiral, a spiral that combines the focusing properties of a FZP and the phase dislocation of spiral gratings, allowing 
the generation of strongly focused vortex beams. The constructive interference between open and closed zones in the grating, in analogy with the FZP lens, allows theoretically and experimentally sharp beam focusing, enhancing the field intensity at the focus up to 170 times. The spiral shape of the grating imposes the helicity, rotating the phase of the diffracted waves and creating a phase dislocation along the axis. We show that these Fresnel-spiral diffraction gratings produce geometrically optimal focusing of the beams if compared with other spiral curves, as for instance the Fermat's Archimedean curve. In this way, all diffracted waves by the present grating geometrically converge to the same focal plane. In particular, the system provides a tunable topological charge of the vortex beam by using different numbers of arms in the Fresnel-spiral diffraction grating, since the topological charge is equal to the number of arms. Two different Fresnel-spiral diffraction gratings with topological charges of 1 and 5 are experimentally tested showing excellent agreement with the theory and simulations.

We start by the definition of the Fresnel-spiral diffraction grating that consists of a set of spiral arms, which alternate between opaque and transparent. In general, the Fresnel-spiral diffraction grating can be made of $M$ arms. The position of the opaque spiral arms, i.e., the spiral curve $r(\theta)$ of the $m$-th arm, is given by

$$
r_{m}(\theta)^{2}=\left[\sqrt{r_{0}^{2}+F^{2}}+\left(\frac{M \theta}{2 \pi}-m\right) \lambda\right]^{2}-F^{2},
$$

where $F$ is the focal distance of the lens, $\lambda$ is the wavelength, and $r_{0}^{2}=(F+\lambda)^{2}-F^{2}$ is the radius of the central opaque area. We notice that for the particular case with a single arm $(M=m=1)$, Eq. (1) reproduces the classical FZP expression if each angular turn is substituted by a discrete sequence of annular rings, $\theta=2 n \pi$, i.e., $r(n)^{2}=\left(\sqrt{r_{0}^{2}+F^{2}}+n \lambda\right)^{2}$ $-F^{2}$ with $n=0,1,2, \ldots$ Therefore, the Fresnel-spiral diffraction grating can be understood as a FZP where the grating lines are twisted into a spiral shape. In a classical FZP, waves irradiating the zone plate diffract from the opaque zones and constructively interfere at the desired focus. However, in the case of a Fresnel-spiral grating, waves diffracted from opposite angles arrive at the focus in the opposite phase due to the fact that the path difference between both trajectories is $\lambda / 2$, as schematically shown in Figs. 1(a) and 1(b). Then, in the focal plane, the destructive interference leads to the generation of a hollow focused beam.

Figure 1(c) shows a Fresnel-spiral zone plate focusing at $F=45 \lambda$ (blue continuous line). We notice that there is a perfect match with the radius of the annular areas of equivalent FZP rings (open circles) at $\theta=2 \pi n$. The geometrical focal distance of the diffracted waves of an axisymmetric grating can be calculated as $F_{n}^{2}=\left[\left(r_{n+1}^{2}-r_{n}^{2}\right) / 2 \lambda-\lambda / 2\right]^{2}$ $-r_{n}^{2}$. Thus, Fig. 1(d) shows the geometrical focus of the locally diffracted waves by the spiral by setting $r_{n}=r(\theta)$ and $r_{n+1}=r(\theta+2 \pi)$ for Fresnel-spiral zone plate focusing at $F=45 \lambda$. We can observe that for all the areas of the Fresnel spiral, i.e., for all the spiral windings, the geometrical focal distance remains constant and matches the equivalent FZP. In order to compare with the different spiral profiles used in the literature, we have calculated and compared the geometrical distribution and the focal spots of an (a)
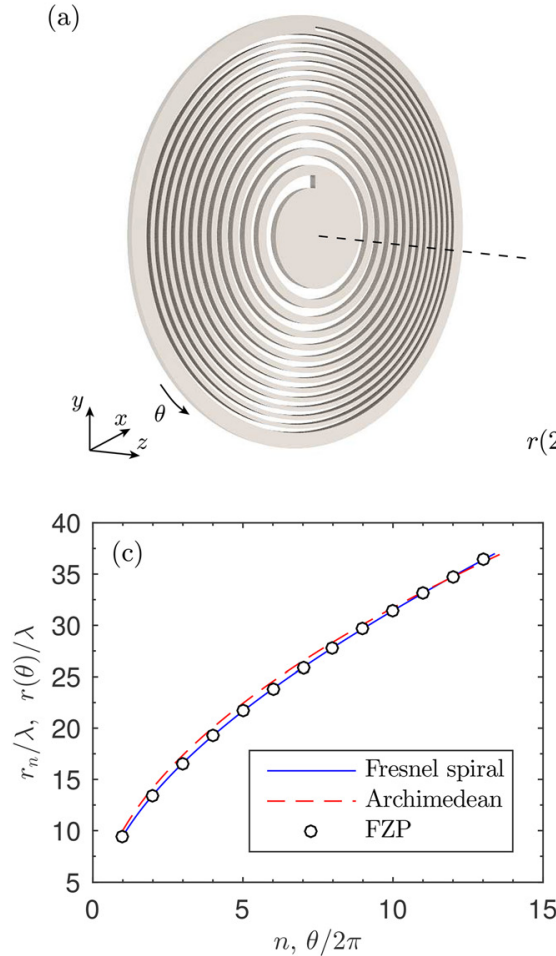

(b)
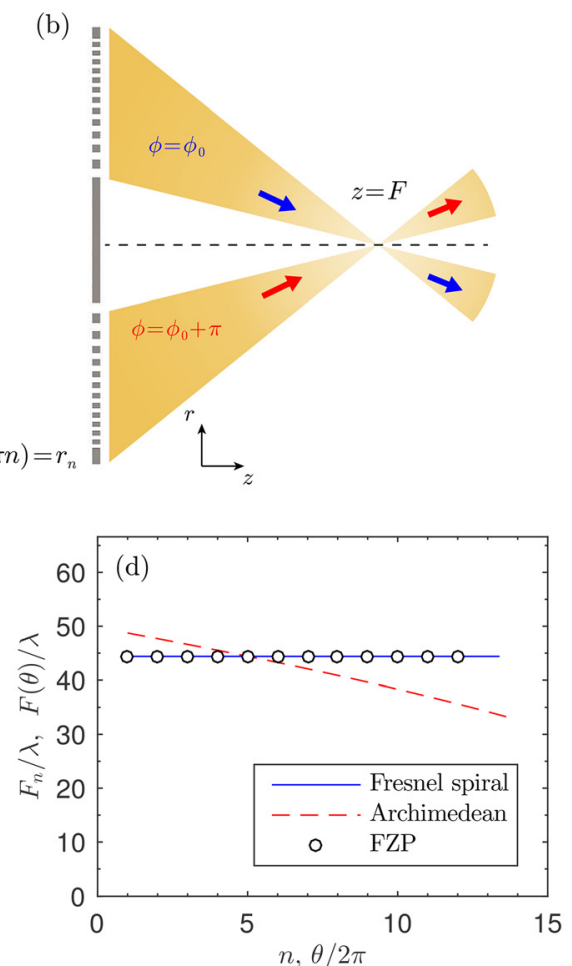
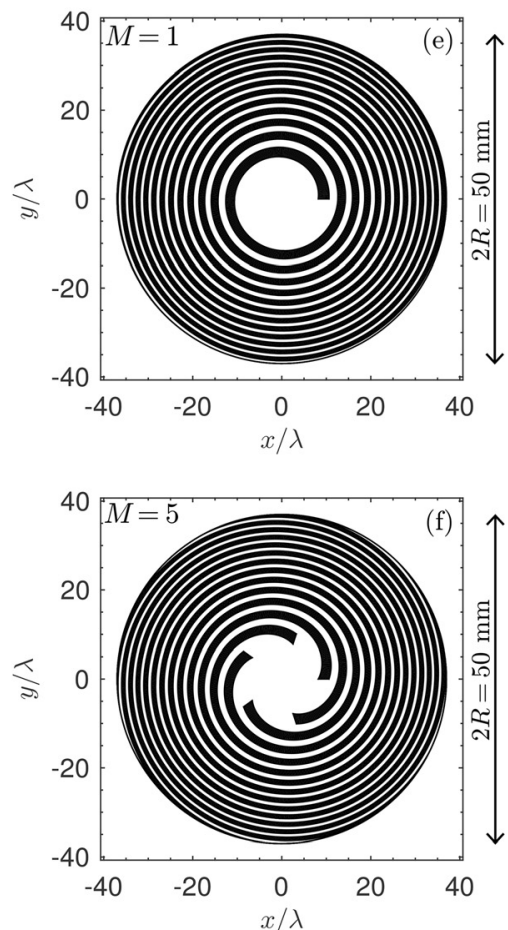

FIG. 1. (a) Scheme of a Fresnel spiral zone plate with $M=1$ arms. (b) Cross-section of the Fresnel-spiral zone plate at $(x=0)$ showing the Fresnel zone plate profile. (c) Polar curve for the design of a Fresnel-spiral zone plate (continuous blue) focusing at $F=45 \lambda$, Archimedean spiral with $\gamma=0.5$ (dashed red), and radius of the FZP rings (circles). (d) Geometrical focal distance calculated for the Fresnel-spiral zone plate (continuous blue), Archimedean spiral with $\gamma=0.5$ (dashed red), and FZP rings (circles). (e) Scheme of the zone plates used in the experiments for $M=1$ and (f) $M=5$. 
Archimedean spiral. An Archimedean spiral curve is given by $r(\theta)=a \theta^{\gamma} / 2 \pi$, where $a$ is the radial separation of the annular scatters between windings, i.e., the radial grating step, and $\gamma$ is an exponent which defines the growth rate of the radial grating step. The Archimedean spiral with $\gamma=0.5$ (Fermat spiral curve) apparently matches the Fresnel-spiral, as shown in Fig. 1(c). However, the Archimedean spiral produces strong variations of the focal position of the hollow spot [red dashed line in Fig. 1(d)], leading to focusing aberrations. Therefore, the hollow focused beam produced by the Fresnel-spiral grating is geometrically optimal. A detailed analysis of the focusing performance of Archimedean spiral gratings with different $\gamma$ is given in the supplementary material. The results show that special attention should be paid when designing Archimedean-spiral diffraction gratings.

To validate the theoretical designs, two Fresnel-spiral gratings were constructed for the ultrasound regime, with $M=1$ and $M=5 \mathrm{arms}$, both for a design frequency of $f=2.220 \mathrm{MHz}$, a focal length of $F=30 \mathrm{~mm}$, and an aperture of the source of $2 R=50 \mathrm{~mm}$, as sketched in Figs. 1(e) and 1(f), respectively. The gratings were manufactured by laser cutting in stainless steel, with a sound speed of $c_{l}=5790 \mathrm{~m} / \mathrm{s}$ and a density of $7480 \mathrm{~kg} / \mathrm{m}^{3}$. For $M=1$, a lens with a total of 14 windings was fabricated, while for $M=5$, each arm winds 3 times. In addition, radial bars of $0.5 \mathrm{~mm}$ thickness were added for robustness, which do not influence the focusing properties. We consider propagation in degassed-distilled water at $20^{\circ} \mathrm{C}$, with a sound speed of $c_{0}=1490 \mathrm{~m} / \mathrm{s}$ and a density of $998 \mathrm{~kg} / \mathrm{m}^{3}$. The source was driven by a 50 cycle sinusoidal pulse burst at the design frequency using a function generator (14 bits, $100 \mathrm{MS} / \mathrm{s}$, model PXI5412, National Instruments) and a linear amplifier (ENI $1040 \mathrm{~L}, 400 \mathrm{~W}, 55 \mathrm{~dB}$, ENI, Rochester, NY). The pressure waves were recorded by a HNR $500 \mu \mathrm{m}$ needle polymer polyvinylidene fluoride hydrophone (Onda Corporation, CA), and a digitizer (64 MS/s, model PXI5620, National Instruments) was used. A three-axis micro-positioning system (OWIS GmbH, Germany) was used to move the hydrophone in three orthogonal directions with an accuracy of $10 \mu \mathrm{m}$, and a National Instruments PXI-Technology controller NI8176 was used to control all the devices. The gratings were mounted above the piezoelectric flat source as Fig. 2(a) shows, at a distance of $0.7 \mathrm{~mm} \approx \lambda$; therefore, one observes small aberrations in the beam due to the cavity resonances. Note that the corresponding wavelength in water is $0.67 \mathrm{~mm}$.

Assuming the solid material of the spiral as a perfect opaque area, the transmitted field can be estimated theoretically by the Rayleigh-Sommerfeld diffraction integral. ${ }^{20}$ The acoustic pressure fields obtained theoretically and experimentally are shown in Fig. 2(b), with $p$ being the pressure field and $p_{0}$ the pressure at the source plane. Perfect agreement is observed in the field distribution. Due to the relatively low impedance contrast between the steel plate and the water, waves in the experiment are allowed to propagate in the bulk of the plate. Thus, we developed a full-wave numerical simulation using the pseudo-spectral time domain method with a $k$-space corrector, ${ }^{29}$ whose results are also shown in Fig. 2(b). Excellent agreement between theory and simulation was obtained, suggesting that the impedance contrast of the steel/water is sufficient to generate diffraction grating effects. The axial distribution of the acoustic intensity is shown in Fig. 2(c), showing a total intensity enhancement of 170 times with respect to the intensity of the source $(22.3 \mathrm{~dB})$. Excellent agreement was found between all methods. The field cross-section distributions are given in Figs. 2(d)-2(g). First, the phase of the field (d) and (e) shows the characteristic screw dislocation, with a topological charge of $M=1$. Then, the magnitude of the field (f) and ( $\mathrm{g}$ ) clearly
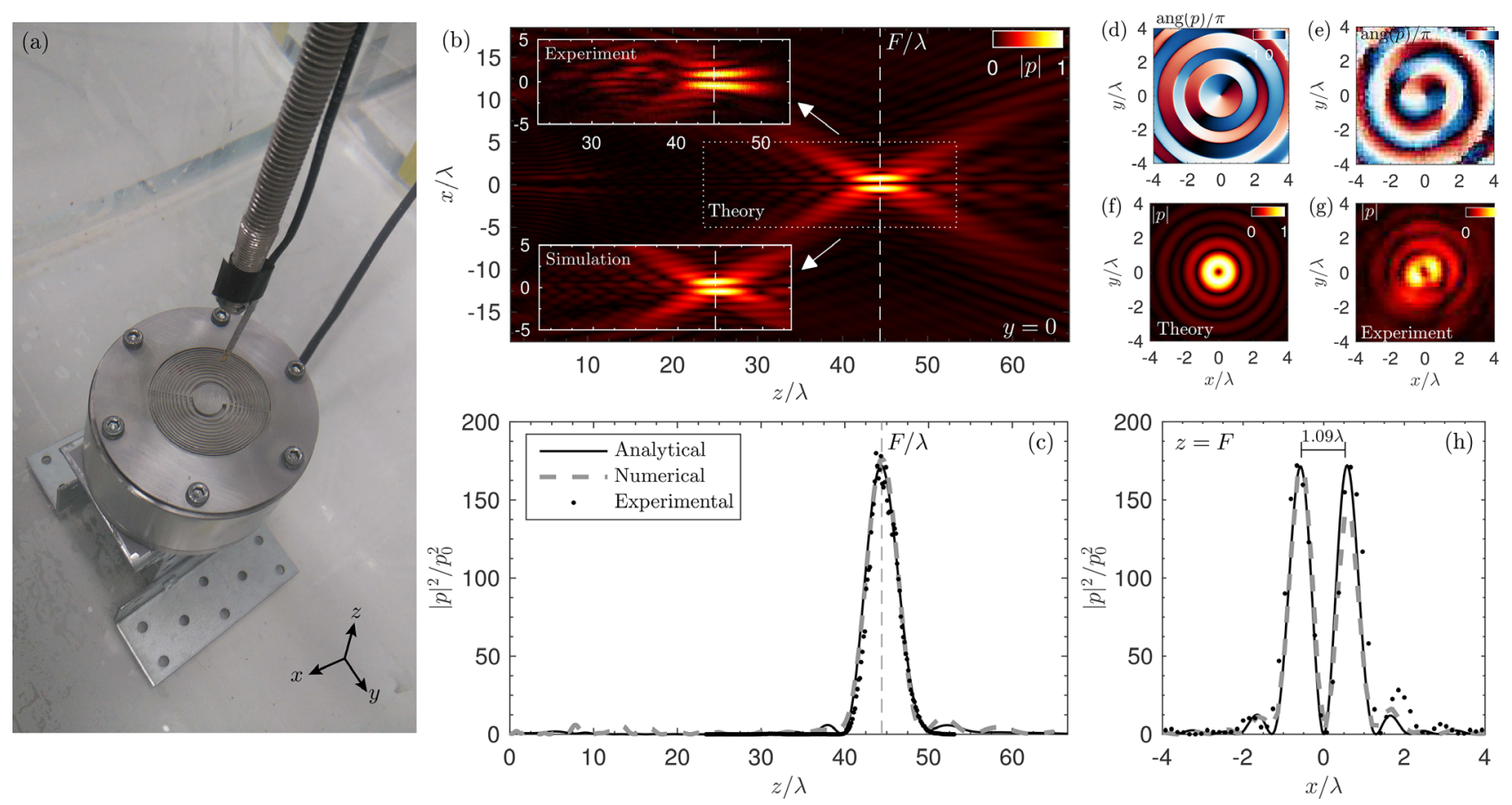

FIG. 2. (a) Photograph of the set-up for the $M=1$ Fresnel-spiral grating. (b) Theoretical, experimental, and simulated field cross-section in the plane $y=0$. The geometrical focal distance is shown with the dashed line. (c) Axial distribution of the intensity measured at $y=0$ and $x / \lambda=0.58$. Theoretical and experimental transversal field distributions at $z=F$, (d) and (e) phase and (f) and (g) magnitude. (h) Transversal intensity distribution measured at $y=0$ and $z=F$. See supplementary material video for time-domain field representations. 

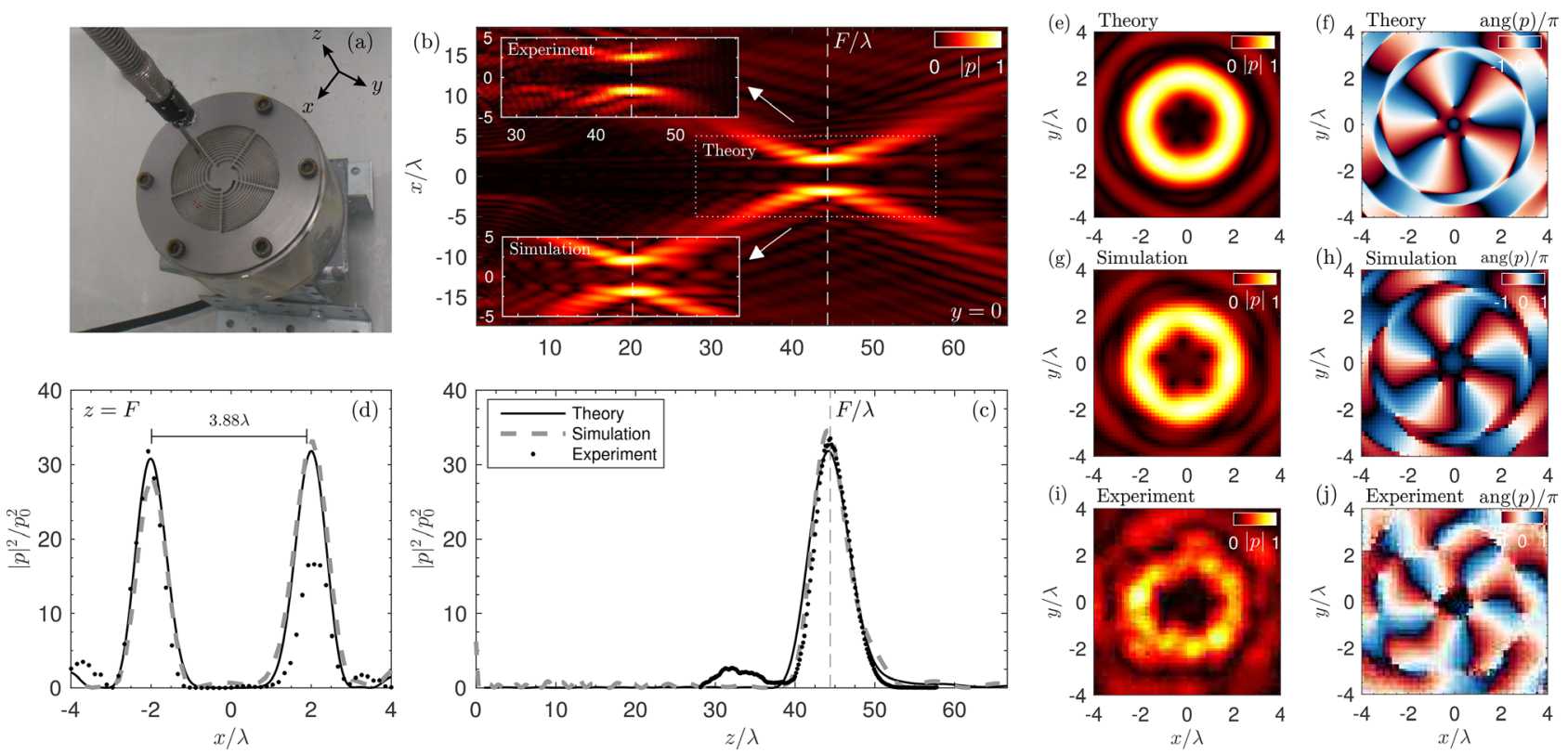

FIG. 3. (a) Photograph of the set-up for the $M=5$ Fresnel-spiral grating. (b) Theoretical, experimental, and simulated field cross-section in the plane $y=0$. (c) Axial distribution of the intensity measured at $y=0$ and $x / \lambda=2.03$. (d) Transversal intensity distribution measured at $z=F$ and $y=0$. Theoretical, simulated, and experimental transversal field distributions at $z=F$, (e), (g), and (i) magnitude and (f), (h), and (j) phase. See supplementary material video for a time-domain field representation.

shows the null at the axis characteristic of the highly focused vortex beam. Some lateral side lobes are observed due to diffraction effects. The time time-domain field representation is given in the supplementary material video.

Figure 3 shows the corresponding results for the case of a higher topological charge spiral plate. The experimental set-up is shown in Fig. 3(a), while the measured field is shown in Fig. 3(b). We observe that for high-topological charge vortices, the designed structure efficiently focuses the beam, while the null at the focal spot widens. The intensity distribution, Fig. 3(c), shows that the gain of the system decreases with the topological charge: note that if the area covered by the wide toroidal focus is higher, then the energy density decreases. This is observed in more detail in the transverse field shown in Fig. 3(d), while the spatial information is given in the maps (e)-(j). The transversal field distributions show the characteristic ring like structure of high order Bessel beams. A screw dislocation is observed at the axis, with a null of the field on the axis. The topological charge of the vortex, which can be measured from the phase distributions (f), (h), and (j), corresponds to the number of arms of the spiral, $M=5$. The time time-domain field representation is given in the supplementary material video.

Finally, an estimation of the size of the central hollow area is marked in Figs. 2(h) and 3(d). Note that in the specific case of high-order Bessel beams, the size of the hollow part can be estimated analytically as a function of the topological charge $M$ as $d_{\text {Bessel }} \approx\left(M+0.8086 M^{1 / 3}\right) / k_{r}$, with $k_{r}$ being the radial component of the wave-vector. ${ }^{20}$ For a Bessel beam extending from $z=0$ to $z=F=30 \mathrm{~mm}$, with a total aperture of $2 R=50 \mathrm{~mm}$, we obtain $k_{r}=6.1161 \times 10^{3} \mathrm{~m}^{-1}$. Therefore, for the single-arm spiral $(M=1)$, the width of the hollow areas is $d_{\text {Bessel }} \approx 0.88 \lambda$ and $d_{\text {Fresnel }} \approx 1.09 \lambda$ for the Bessel beam and for the Fresnel-spiral zone plate, respectively. In addition, for the multiple arm-spiral $(M=5)$, we obtain $d_{\text {Bessel }}$ $\approx 3.1 \lambda$ and $d_{\text {Fresnel }} \approx 3.7 \lambda$. Despite the differences, the size of the deaf central core of both the Fresnel-spiral vortex and high-order Bessel beams increases with the topological charge, and a rough estimation can be performed.

In conclusion, in this work, we have shown that the Fresnel-spiral curve is the geometrically optimal structure to design binary diffraction-gratings producing sharp vortex focusing. The designs have been validated experimentally using acoustic waves in the ultrasound regime, where excellent agreement has been found between theory, simulations, and experiments. The designed plates allow sharp acoustic focusing, while the topological charge of the vortex beam can be directly related to the number of arms of the Fresnel-spiral. Note that focusing is produced by diffraction grating; therefore, the separation between opaque and transparent areas should be of the order of half wavelength. Thus, the product between the wavenumber and the size of the lens, $k R$, should be high enough to allow for various windings. This, in principle, can be viewed as a drawback. In fact, using metasurfaces with subwavelength dimensions, acoustical vortices can be focused in the same manner with lower $k R$ ratios. However, if the ratio $k R$ is small, the focusing performance of any focused source is also going to be limited for any lens, except those structures using the evanescent field, as diffraction effects depend on the quantity $k R$. One important final remark is that using any diffraction grating, half of the impinging energy converges to the focal spot while other half diverges away from the axis. In this way, only half of the impinging energy on a Fresnel-Spiral Zone Plate can be focused. The results given in this paper pave the road to construct wave-matter interaction systems using simple and passive devices, as those required to construct acoustic particle manipulation and trapping systems.

See supplementary material for (1) the time-domain field representation (video) of a single-topological charge vortex beam, (2) the time-domain field representation of the 
$M=5$ topological charge vortex beam, and (3) an extended analysis of the focusing performance of Archimedean spiral gratings with different $\gamma$.

This work was supported by the Spanish Ministry of Economy and Innovation (MINECO) and European Union FEDER through Project Nos. FIS2015-65998-C2-1 and FIS2015-65998-C2-2. N.J. acknowledges financial support from Generalitat Valenciana through Grant No. APOSTD2017-042.

${ }^{1}$ J. Nye and M. Berry, Proceedings of the Royal Society of London A: Mathematical, Physical and Engineering Sciences (The Royal Society, 1974), Vol. 336, pp. 165-190.

${ }^{2}$ D. G. Grier, Nature 424, 810 (2003).

${ }^{3}$ K. Volke-Sepúlveda, A. O. Santillán, and R. R. Boullosa, Phys. Rev. Lett. 100, 024302 (2008).

${ }^{4}$ A. Anhäuser, R. Wunenburger, and E. Brasselet, Phys. Rev. Lett. 109, 034301 (2012).

${ }^{5}$ C. E. Demore, Z. Yang, A. Volovick, S. Cochran, M. P. MacDonald, and G. C. Spalding, Phys. Rev. Lett. 108, 194301 (2012).

${ }^{6}$ Z. Hong, J. Zhang, and B. W. Drinkwater, Phys. Rev. Lett. 114, 214301 (2015).

${ }^{7}$ J. Wu, J. Acoust. Soc. Am. 89, 2140 (1991).

${ }^{8}$ A. Marzo, A. Ghobrial, L. Cox, M. Caleap, A. Croxford, and B. Drinkwater, Appl. Phys. Lett. 110, 014102 (2017).

${ }^{9}$ A. Marzo, M. Caleap, and B. W. Drinkwater, Phys. Rev. Lett. 120, 044301 (2018).

${ }^{10}$ C. Shi, M. Dubois, Y. Wang, and X. Zhang, Proc. Natl. Acad. Sci. U. S. A. 114, 7250 (2017).

${ }^{11}$ J.-L. Thomas and R. Marchiano, Phys. Rev. Lett. 91, 244302 (2003).
${ }^{12}$ R. Marchiano and J.-L. Thomas, Phys. Rev. E 71, 066616 (2005).

${ }^{13}$ J. L. Ealo, J. C. Prieto, and F. Seco, IEEE Trans. Ultrason. Ferroelectr. Freq. Control 58, 1651 (2011).

${ }^{14}$ S. Gspan, A. Meyer, S. Bernet, and M. Ritsch-Marte, J. Acoust. Soc. Am. 115, 1142 (2004).

${ }^{15}$ B. T. Hefner and P. L. Marston, J. Acoust. Soc. Am. 106, 3313 (1999).

${ }^{16}$ X. Jiang, Y. Li, B. Liang, J.-C. Cheng, and L. Zhang, Phys. Rev. Lett. 117, 034301 (2016).

${ }^{17}$ L. Ye, C. Qiu, J. Lu, K. Tang, H. Jia, M. Ke, S. Peng, and Z. Liu, AIP Adv. 6, 085007 (2016).

${ }^{18}$ C. J. Naify, C. A. Rohde, T. P. Martin, M. Nicholas, M. D. Guild, and G. J. Orris, Appl. Phys. Lett. 108, 223503 (2016).

${ }^{19}$ H. Esfahlani, H. Lissek, and J. R. Mosig, Phys. Rev. B 95, 024312 (2017).

${ }^{20}$ N. Jiménez, R. Picó, V. Sánchez-Morcillo, V. Romero-García, L. M. García-Raffi, and K. Staliunas, Phys. Rev. E 94, 053004 (2016).

${ }^{21}$ T. Wang, M. Ke, W. Li, Q. Yang, C. Qiu, and Z. Liu, Appl. Phys. Lett. 109, 123506 (2016).

${ }^{22}$ R. D. Muelas-Hurtado, J. L. Ealo, J. F. Pazos-Ospina, and K. VolkeSepúlveda, Appl. Phys. Lett. 112, 084101 (2018).

${ }^{23}$ X. Jiang, J. Zhao, S.-L. Liu, B. Liang, X.-Y. Zou, J. Yang, C.-W. Qiu, and J.-C. Cheng, Appl. Phys. Lett. 108, 203501 (2016).

${ }^{24}$ N. Jimenez, V. Romero-García, R. Picó, A. Cebrecos, V. J. SánchezMorcillo, L. Garcia-Raffi, J. V. Sánchez-Pérez, and K. Staliunas, Europhys. Lett. 106, 24005 (2014).

${ }^{25}$ L. Sanchis, A. Yánez, P. L. Galindo, J. Pizarro, and J. M. Pastor, Appl. Phys. Lett. 97, 054103 (2010).

${ }^{26}$ S. Farnow and B. Auld, Appl. Phys. Lett. 25, 681 (1974).

${ }^{27}$ M. Molerón, M. Serra-Garcia, and C. Daraio, Appl. Phys. Lett. 105, 114109 (2014).

${ }^{28}$ N. Jimenez, V. Romero-García, R. Picó, L. M. Garcia-Raffi, and K. Staliunas, Appl. Phys. Lett. 107, 204103 (2015).

${ }^{29}$ B. T. Cox, S. Kara, S. R. Arridge, and P. C. Beard, J. Acoust. Soc. Am. 121, 3453 (2007). 